\title{
Decentralized AP Selection in Large-Scale Wireless LANs Considering Multi-AP
}

\section{Interference}

\author{
Phillip B. Oni and Steven D. Blostein Department of Electrical and Computer \\ Engineering \\ Queen's University, Kingston, ON, Canada. \\ Email: \{phillip.oni, steven.blostein \}@queensu.ca
}

\begin{abstract}
Densification of access points (APs) in wireless local area networks (WLANs) increases the interference and the contention domains of each AP due to multiple overlapped basic service sets (BSSs). Consequently, high interference from multiple co-channel BSS at the target AP impairs system performance. To improve system performance in the presence of multi-BSSs interference, we propose a decentralized AP selection scheme that takes interference at the candidate APs into account and selects AP that offers best signal-interference-plus noise ratio (SINR). In the proposed algorithm, the AP selection process is distributed at the user stations (STAs) and is based on the estimated SINR in the downlink. Estimating SINR in the downlink helps capture the effect of interference from neighboring BSSs or APs. Based on a simulated large-scale 802.11 network, the proposed scheme outperforms the strongest signal first (SSF) AP selection scheme used in current 802.11 standards as well as the mean probe delay (MPD) AP selection algorithm in [3]; it achieves $99 \%$ and $43 \%$ gains in aggregate throughput over SSF and MPD, respectively. While increasing STA densification, the proposed scheme is shown to increase aggregate network performance.
\end{abstract}

\section{Index Terms}

wireless LANs, access points, AP selection, dense deployments 


\section{INTRODUCTION}

The popularity of IEEE 802.11 or Wireless Fidelity (Wi-Fi) networks among users as an affordable data access network is increasing tremendously, and consequently, causing an increase in the number of access points (APs) deployed in places like residential/apartment buildings, hotels, airports, campuses and enterprise buildings. The unprecedented demand for affordable high data rate and the emergence of bandwidth intensive applications is also a contributing factor. Similarly, the emerging cellular-WiFi offloading trend requires a high density of APs to handle the huge mobile data traffic [1]. With this promising solution to explosive mobile data traffic comes increased inter-AP interference, which degrades the capacity of dense wireless local area networks (DWLANs).

Although densification of APs provides extended coverage and affordable data access in homes, offices and campuses, deploying large numbers of APs over a confined network area increases the interference domain of each AP and causes severe interference to neighboring APs. This becomes worrisome in cases where AP cells overlap leading to overlapped basic service sets (OBSS) where inter-BSS or inter-AP interference becomes significant [2]; a BSS consists of an AP and its associated stations (STAs). In addition to increasing AP's interference domain, uncoordinated distribution of STAs among APs causes overwhelming channel access contention at overloaded cells due to the carrier sense multiple access collision avoidance (CSMA/CA) protocol specified in the IEEE 802.11 standard for channel acquisition. Interference, data collisions and congestion are major concerns in DWLAN.

One technique to improve system performance in the presence of multiple interference sources is to coordinate AP selection based on key performance metrics such as SINR and packet error rate (PER). Currently, the AP selection process in WLAN is based on the strongest received signal strength (RSS) or strongest signal first (SSF), a method whereby an STA selects AP that offers strongest RSS without considering interference, congestion and load at the candidate AP. This legacy AP selection scheme defined in the 802.11 standard might cause a high degree of contention in some BSSs, and consequently degrades aggregate network performance. Studies [3] - [10] focus on proposing new schemes for AP selection in 802.11 networks and demonstrate the inability of an RSS-based scheme to guarantee a level system performance. 


\section{Existing AP SELECTION SCHEMES}

In [3] and [4], authors focus primarily on achieving a fair distribution of STAs among APs to achieve load balancing as opposed to the SSF AP selection scheme that has the tendency to cause load imbalances among APs [9]. The probe delay (PD) and mean probe delay (MPD) algorithms [3] select the AP with minimum probe delay. Similarly, an AP association control scheme is proposed in [4] to achieve proportional fairness. A graph matching approach to coordinate AP association and maximize uplink throughput is proposed in [5], where the links between STAs and APs are modeled as graph edges with uplink SINRs as edge weights. In [6], virtualizing wireless network interfaces enables STAs to associate with multiple APs and switch between APs without severe overhead thereby making the AP selection dynamic.

In [7], by introducing a Quality of Service (QoS) differentiated information element (IE) in frames advertised by APs, STAs are aware of the call blocking probability when selecting an AP. By measuring channel utilization, the authors in [8] proposed an AP selection scheme that allows STAs to select AP with minimum hidden terminal effect. Similarly, using a channel measurement approach, the authors in [9] suggest that the hidden terminal problem and frame aggregation are factors in selecting an AP that guarantees better throughput. Another measurement-based AP selection approach is presented in [10] using a supervised learning technique (Multi-Layer Feed-Forward Neural Network) with multiple inputs, which allows STAs to select the AP that offers best performance.

\section{CONTRibution}

Thus far, inter-BSS interference at the target AP has not been considered when selecting AP. The proposed AP selection scheme exploits awareness of inter-AP interference by allowing STAs to estimate the received SINRs from a set of candidate APs and select an AP with the best SINR. The goal is to improve system performance by associating STAs with APs that offer best SINR. Enhancing performance in the presence of inter-AP interference is important in DWLANs

for two reasons. First, the problem of OBSS is inevitable in large-scale dense AP deployments, leading to severe inter-AP interference, which degrades performance due to close proximity of co-channel APs.

Second, the majority of the traffic is in the downlink. Taking video streaming as an example, after a user requests the service in the UL, the entire video streaming session occurs in the 
downlink. The remaining parts of this paper are organized as follows. First, we present the system and network model in Section IV. The proposed scheme is presented in Sections $\mathrm{V}$ and VI while simulation results are presented in Section VII Section VIII concludes this paper. The summary of key symbol definitions is presented in Table 1 for easy reference.

TABLE I

Key Notations.

\begin{tabular}{|c|c|}
\hline Notation & Definition \\
\hline $\mathcal{S}$ & Set of stations (STAs) \\
\hline $\mathcal{A}$ & Set of access points (APs) \\
\hline$M=|\mathcal{A}|$ & Total number of APs \\
\hline$N=|\mathcal{S}|$ & Total number of STAs \\
\hline$\Gamma$ & Physical carrier sensing (PCS) threshold \\
\hline$\omega$ & A channel from set of orthogonal channels \\
\hline$P^{t}$ & Transmit power \\
\hline $\mathcal{A}^{\omega}$ & Set of co-channel APs on channel $\omega$ \\
\hline $\mathcal{A}_{a}^{\omega}$ & Set of active APs (permitted by CSMA/CA to transmit) \\
\hline $\mathcal{A}^{I}$ & Set of APs interfering \\
\hline $\mathcal{A}_{i}^{c}$ & Set of candidate APs \\
\hline$P_{j i}^{r}$ & Received power by $\mathrm{STA}_{i}$ from $\mathrm{AP}_{j}$ \\
\hline$\gamma_{o}$ & SINR threshold \\
\hline$\theta$ & Receiver sensitivity \\
\hline $\mathcal{I}_{T}^{j i}$ & Total interference power on a desired link \\
\hline$t_{j i}$ & Transmission time of a frame \\
\hline$\Lambda_{j i}$ & Transmission rate \\
\hline $\mathbf{B}_{j i}$ & Expected throughput of a link \\
\hline
\end{tabular}

\section{System Model AND PROBLEM Formulation}

In this section, the system model for a DL (AP-to-STA) transmission is presented. In the downlink (DL) of a WLAN, APs transmit data to their respective associated STAs as shown in our system model in Figure 1. The achievable throughput in the downlink is of particular concern because the majority of dense Wi-Fi traffic will be in the downlink. On a typical DWLAN, let the set of APs be denoted as $\mathcal{A}$ serving a set $\mathcal{S}$ of STAs. Hence, the entire network consists of $M=|\mathcal{A}|$ APs and $N=|\mathcal{S}|$ STAs. For this downlink model, we will assume that all APs transmit 
at the same power $P^{t}(\mathrm{~mW})$ and $d_{j i}(\forall j \in \mathcal{A}, i \in \mathcal{S})$ is the distance between the transmitting $\mathrm{AP}_{j}$ and the receiving $\mathrm{STA}_{i}$ as shown in Figure 1 .

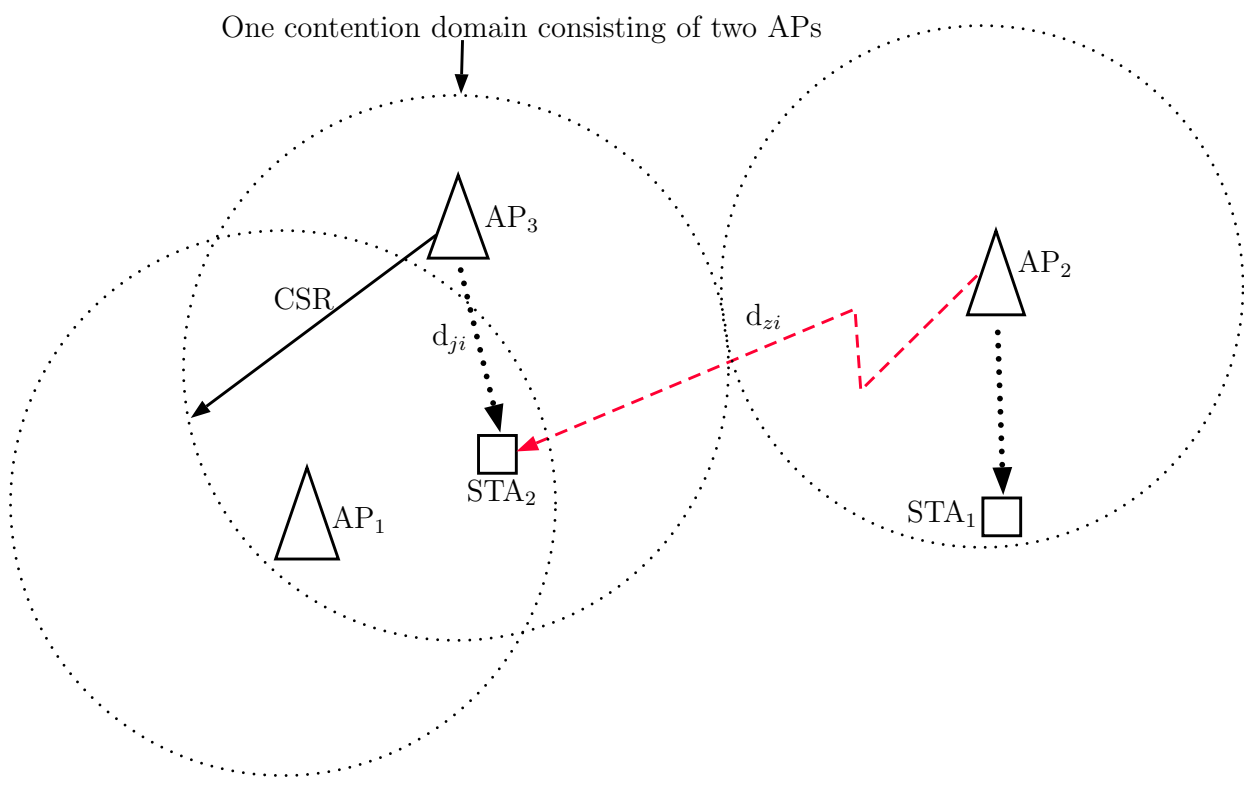

Fig. 1. System and interference model.

Next, we describe channel contention in the downlink of a WLAN. The number of available orthogonal channels in Wi-Fi networks depends on the version of the IEEE 802.11 standard being supported. For instance, the IEEE $802.11 \mathrm{~b} / \mathrm{g}$ standard supports 3 non-overlapping channels from 14 available channels while IEEE 802.11a provides 8 non-overlapping channels. The more recent standard, IEEE $802.11 \mathrm{ac}$, has two non-overlapping channels for $80 \mathrm{MHz}$ and one $160 \mathrm{MHz}$ nonoverlapping channel. Therefore, due to an insufficient number of orthogonal channels for large AP deployments, many APs are deployed on the same channel and some BSSs overlap due to close proximity. Consequently, two or more APs must contend for the same channel before transmitting.

In Figure 1, let $\mathrm{AP}_{3}$ and $\mathrm{AP}_{1}$ represent co-channel APs within carrier sensing range (CSR) of each other. The CSR depends on the clear channel assessment (CCA) threshold used during the physical carrier sensing (PCS) process. PCS is usually performed within the CSR to determine the presence of active transmissions on the channel. In order for an AP to detect the presence of an active AP on the channel, the energy level sensed during PCS is compared to the CCA threshold. The channel is occupied by another AP within the CSR if the sensed energy level 
is greater than the CCA threshold. Therefore, with the PCS process in CSMA/CA, whenever $\mathrm{AP}_{3}$ has the channel for transmission, $\mathrm{AP}_{1}$ remains silent; all co-channel $\mathrm{APs}$ do not transmit concurrently.

For any supported 802.11 standard, let $\omega$ denote a channel belonging to the set of available orthogonal channels. Let us denote the set of co-channel APs on channel $\omega$ as $\mathcal{A}^{\omega}$ and let $\mathcal{A}_{a}^{\omega}$ represent the set of active APs (permitted by CSMA/CA to transmit) in $\mathcal{A}^{\omega}$ on channel $\omega$. By virtue of the CCA threshold, a subset of the active APs in $\mathcal{A}_{a}^{\omega}$ will be in the contention domain (within CSR) of $\mathrm{AP}_{j}, \forall j \in \mathcal{A}$. Therefore, all active APs in the contention domain of $\mathrm{AP}_{j}$ form a set of co-channel APs with $\mathrm{AP}_{j}$ and this set is denoted as $\mathcal{A}_{j}^{\omega}$. This implies that $\mathrm{AP}_{j}$ will contend for the medium with other co-channel APs in $\mathcal{A}_{j}^{\omega}$, and if $\mathrm{AP}_{j}$ or any other $\mathrm{AP}$ in $\mathcal{A}_{j}^{\omega}$ is transmitting on the channel, other APs are idle; mathematically: $\mathcal{A}_{j}^{\omega}:=\left\{m \in \mathcal{A}_{a}^{\omega}, m \neq j \mid \lambda>\right.$ $\left.\Gamma, \mathcal{A}^{\omega} \subseteq \mathcal{A}\right\}, j \in \mathcal{A}, \omega \in \mathcal{C}$, where $\Gamma$ denotes the PCS (or CCA) threshold, $\lambda$ is the signal power sensed on channel $\omega$ during the PCS, and $\mathcal{C}$ is the set of channels in any supported IEEE 802.11 standard. All APs within the CSR of $\mathrm{AP}_{j}$ are not potential interference sources because the CSR area is cleared during PCS and all other APs within the CSR do not transmit while $\mathrm{AP}_{j}$ is active.

Figure 1 illustrates a downlink interference scenario where $\mathrm{AP}_{2}$ is outside the contention domain of $\mathrm{AP}_{3}$. Therefore, a signal coming from $\mathrm{AP}_{2}$ might interfere with downlink transmissions of $\mathrm{AP}_{3}$ at receiver $\mathrm{STA}_{2}$. From this scenario, the total interference at the receiving $\mathrm{STA}_{2}$ in the downlink can be estimated depending on the number of APs transmitting outside the CSR of $\mathrm{AP}_{3}$ and whose signals are received by $\mathrm{STA}_{2}$. Let $\mathcal{A}^{I}$ represent the set of APs interfering with the downlink signal of $\mathrm{AP}_{j}$ at receiver $\mathrm{STA}_{i}$. The total interference received at $\mathrm{STA}_{i}$ from all interfering APs in $\mathcal{A}^{I}$ is given by

$$
\mathcal{I}^{j i}=\sum_{z \in \mathcal{A}^{I}, i \in \mathcal{S}, j \in \mathcal{A} \mid j \neq z, \mathcal{A}^{I} \subset \mathcal{A}_{j}^{\omega} \subset \mathcal{A}} P_{z i},
$$

where $P_{z i}$ is the received signal power at $\mathrm{STA}_{i}$ from the $z^{\text {th }}$ interfering AP at distance $d_{z i}$. This type of interference measurement is based on a one time capture of the signal strength of an interference source. It does not account for the time variations of the wireless channel and signal strength. Also, the frames received from different interfering sources vary in size and each interfering AP might use a different PHY rate for transmission. Therefore, using the passive interference measurement approach in [12], we can reformulate (1) to account for these 
variations as follows:

$$
\mathcal{I}_{T}^{j i}=\frac{1}{T} \sum_{z=1}^{\left|\mathcal{A}^{I}\right|} \sum_{k=1}^{K} \frac{P_{z i} L_{z i}}{R_{z i}}, z \in \mathcal{A}^{I}, i \in \mathcal{S}, j \in \mathcal{A} \mid j \neq z
$$

where $K$ is the number of frames and $L_{z i}$ is the length of each frame in bits received from $z^{\text {th }}$ interferer, $R_{z i}$ is the PHY rate (bps) at which each frame is received and $T$ denotes the measurement period, and can represent a sufficient number of slot times for accurate estimation. As a result of interfering signal power received at $\mathrm{STA}_{i}$ from $\mathrm{APs}$ outside $\mathrm{AP}_{j}$ 's CSR, the $\mathrm{SINR}$ of the link between $\mathrm{AP}_{j}$ and $\mathrm{STA}_{i}$ is given by

$$
\Psi_{j i}=\frac{P_{j i}^{r}}{\left(\mathcal{I}_{T}^{j i}+N_{o}\right) W}, \quad 1 \leq i \leq N, 1 \leq j \leq M,
$$

where $P_{j i}^{r}$ is the received power from $\mathrm{AP}_{j}$ at $\mathrm{STA}_{i}$ over a distance $d_{j i}$ and $W$ is the system bandwidth. The total transmission time of a desired frame of size $\digamma$ from $\mathrm{AP}_{j}$ to $\mathrm{STA}_{i}$ is denoted as

$$
t_{j i}=\frac{\digamma(\text { bits })}{\Lambda_{j i}}
$$

where $\Lambda_{j i}$ is the transmission rate, which is determined by SINR $\Psi_{j i}$ experienced by $\mathrm{STA}_{i}$ when associated with $\mathrm{AP}_{j}$. The mapping or relationship between $\Lambda_{j i}$ and $\Psi_{j i}$ in an 802.11 WLAN is shown in Table III. Denote the expected throughput of $\mathrm{STA}_{i}$ after associating with $\mathrm{AP}_{j}$ as:

$$
\mathbf{B}_{j i}=\frac{1}{t_{j i}}
$$

which is the case provided prolonged transmission time due to retransmission (aftermath of collision) does not occur. However, when a frame from $\mathrm{AP}_{j}$ to $\mathrm{STA}_{i}$ experiences collision, the transmission time is prolonged as thus:

$$
\tilde{t}_{j i}=\mathrm{DIFS}+t_{b f}+t_{j i}+\mathrm{SIFS}+t_{a c k},
$$

where $t_{b f}=\frac{\mathrm{CW}_{\max }}{2} \times$ Slot-time is the backoff time, $t_{a c k}=\frac{1}{r}$ is the time it takes to transmit ACK frame given basic data rate $r$ (e.g. $1 \mathrm{Mbps}$ in an $802.11 \mathrm{~b}$ network) while SIFS and DIFS are time intervals defined in the 802.11 standard. 
TABLE II

SINR REQUIREMENTS FOR DIFFERENT DATA RATES IN 802.11 [4].

\begin{tabular}{|c|c|c|c|c|c|c|c|c|}
\hline$\Psi_{j i}(\mathrm{~dB})$ & $6-7.8$ & $7.8-9$ & $9-10.8$ & $10.8-17$ & $17-18.8$ & $18.8-24$ & $24-24.6$ & $>24.6$ \\
\hline$\Lambda_{j i}(\mathrm{Mbps})$ & 6 & 9 & 12 & 18 & 24 & 36 & 48 & 54 \\
\hline
\end{tabular}

\section{AP SELECtion Algorithm}

In this section, we present the proposed AP selection method as Algorithm 1. The probe request and probe response frames defined in IEEE 802.11 standards are used to perform interference measurement in the downlink. A typical STA ${ }_{i}$ captures the beacon frames (through channel scanning) from all APs within range to determine the set of candidate APs, $\mathcal{A}_{i}^{c}$, and selects the best-serving AP. Let $\kappa$ be the set of APs within range of $\mathrm{STA}_{i}$. In Step 2, a typical $\mathrm{STA}_{i}$ listens to beacon frames from all APs within range and sorts the RSSs of the beacon frames in decreasing order (Step 3) and selects the AP with best RSS to complete SSF association.

The SSF association is important to ensure that all STAs can discover APs within range and continue to transmit/receive payloads while estimating SINRs from all candidate APs. This prevents starvation in cases when a STA cannot find the AP offering best SINR. Once SSF association is achieved by $\mathrm{STA}_{i}$, it proceeds in Step 4 to create set $\mathcal{A}_{i}^{c}$ of candidate APs for $\mathrm{STA}_{i}$ from the set of APs within range. An AP is added to $\mathcal{A}_{i}^{c}$ if its RSS at $\mathrm{STA}_{i}$ satisfies the minimum receiver sensitivity constraint i.e., $P_{j i}^{r}>\theta$. The choice of $\theta$ depends on the supported data rate. For example, in a typical 802.11 network, to support a minimum data rate of 12 Mbps, the receiver sensitivity $\theta$ is $-79 \mathrm{dBm}$ (for successful reception). After obtaining the set of candidate APs, the channel measurement using probe request and response frames begins in Step 5 .

The typical $\mathrm{STA}_{i}$ sends a directed probe request frame to each $\mathrm{AP}$ in $\mathcal{A}_{i}^{c}$. To increase the accuracy of the interference measurement in Step 5, the algorithm requires that multiple P_RES frames be received by $\mathrm{STA}_{i}$ over a specified period of time. To do this, we define a measurement period $n \times$ Slot-time where $n$ is an integer. Due to the power constraint of most STAs and other low power devices e.g., WiFi-enabled Internet of things (IoTs), Algorithm 1 requires that STAs send only one $\mathrm{P} \_\mathrm{REQ}$ frame but informs candidate $\mathrm{AP}_{j}$ to send multiple $\mathrm{P}_{-} \mathrm{RES}$ frames within $n \times$ Slot-time. The RequestInformation (dot11RadioMeasurementActivated $=$ 


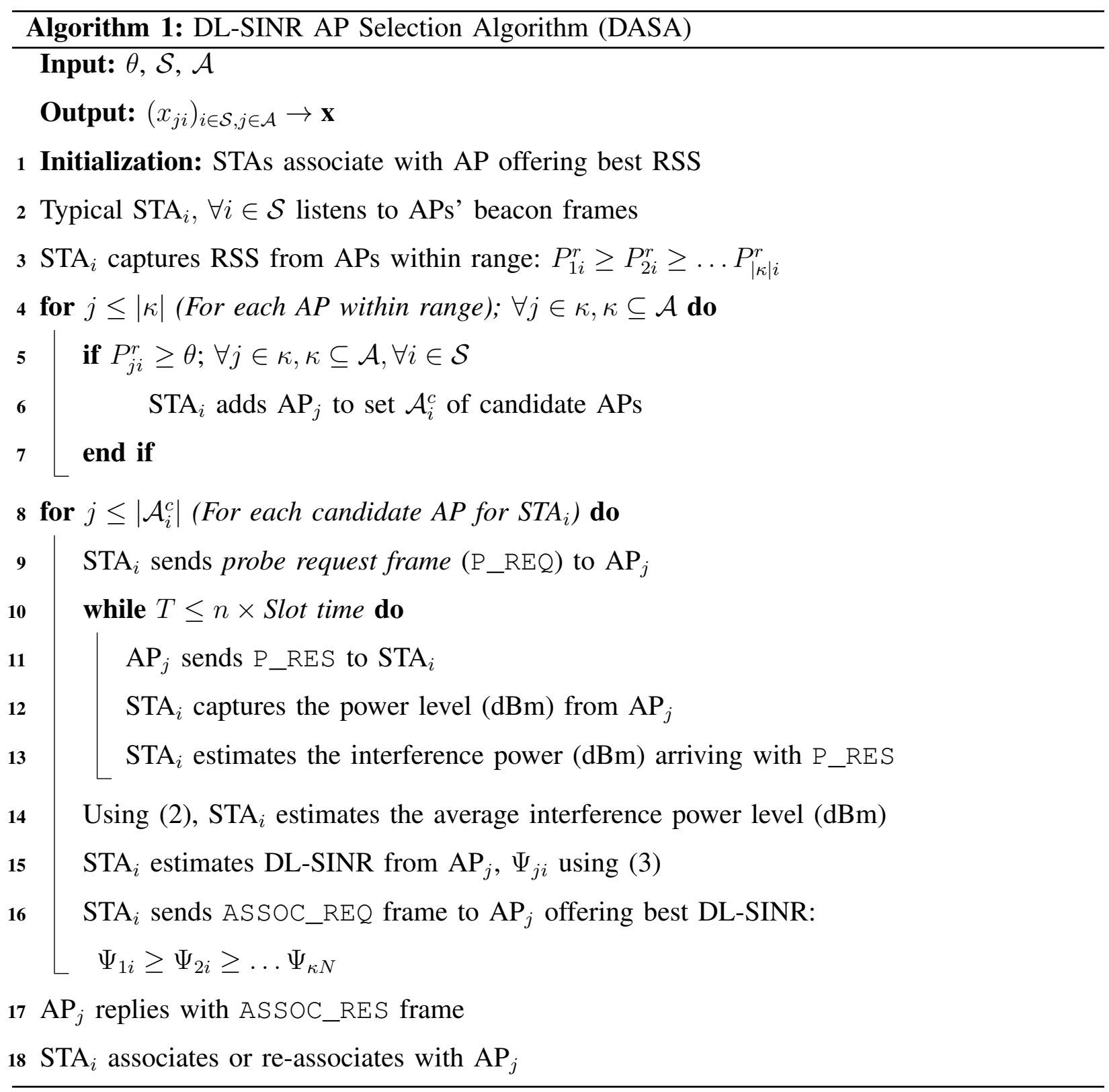

true) parameter of the MLME-Scan.request primitive defined for channel scanning in IEEE 802.11 standard (2012) can be used to inform a candidate $\mathrm{AP}_{j}$ to send multiple $\mathrm{P} \_\mathrm{RES}$ frames.

On receiving the probe response frame ( $\mathrm{P}_{-} \mathrm{RES}$ ) from $\mathrm{AP}_{j}, \mathrm{STA}_{i}$ estimates the DL-SINR based on the magnitude of captured interference power received concurrently with P_RES. Alternatively, this SINR estimation can be done through channel sounding by sending preamble frames or training symbols to candidate APs. Then, it sends association request (ASSOC_REQ) 
frame to the candidate AP that offers the best DL-SINR. The candidate AP responds with association response (ASSOC_RES) frame. This algorithm is easy to implement and does not require modification to 802.11 management frames. Also, we remark that channel measurement capability is available in $802.11 \mathrm{k}$-enabled nodes.

\section{Optimal AP Selection Algorithm}

The AP selection scheme in Section $\mathrm{V}$ might not be optimal under SINR, receiver sensitivity and CCA threshold constraints. These constraints are related to interference distribution across the network. In this section, the problem of AP selection to maximize aggregate throughput is formulated as follows:

$$
\begin{array}{ll}
\text { maximize } & \sum_{j=1}^{M} \sum_{i=1}^{S} \mathbf{B}_{j i} x_{i j} \\
& \sum_{j=1}^{M} x_{i j}=1, \forall i \in \mathcal{S} \\
\text { subject to } & x_{i j} P_{j i}^{r} \geq \theta \quad \forall j \in \mathcal{M} \\
& x_{i j} \Psi_{j i} \geq \gamma_{o} \quad \forall i \in \mathcal{S} \\
& x_{i j} P_{j i}^{c} \leq \Gamma \quad \forall i \in \mathcal{S},
\end{array}
$$

where $x_{i j} \in\{0,1\}, i \in \mathcal{S}, j \in \mathcal{A}, P_{j i}^{c}$ is the total power sensed on the channel during PCS, $\gamma_{o}$ is the SINR threshold, constraint (7b) ensures that each STA associates with only one AP; $x_{i j}=1$ if $\mathrm{STA}_{i}$ is associated with $\mathrm{AP}_{j}$ and $x_{i j}=0$ if otherwise. (7c) is the receiver sensitivity constraint while (7d) and (7e) are the SINR and CCA threshold constraints, respectively. An AP begins transmission if (7e) is satisfied during carrier sensing. This occurs when the total interference power $P_{j i}^{c}$ received from other interfering APs does not exceed $\Gamma$. Therefore, $P_{j i}^{c}=\mathcal{I}^{j i}$ and SINR $\Psi_{j i}$ is related to $\mathcal{I}^{j i}$ by $(3)$. Therefore, any feasible $\left(\Psi_{j i}\right)_{i \in \mathcal{N}, j \in \mathcal{A}}$ that satisfies $7 \mathrm{~d}$ ) also satisfies $(7 \mathrm{e})$, hence, constraint $7 \mathrm{e}$ ) becomes redundant. Similarly, since $P_{j i}^{r}$ and $\Psi_{j i}$ are also related by (3) and $\mathrm{STA}_{i}$ selects $\mathrm{AP}_{j}$ offering best $\mathrm{SINR}$, i.e,

$$
\begin{aligned}
& j^{\prime}=\underset{j}{\arg \min } \mathcal{I}_{T}^{j i} \\
& j^{*}=\underset{j}{\arg \max } \Psi_{j i} \quad
\end{aligned}
$$


TABLE III

SIMULATION PARAMETERS

\begin{tabular}{ll|ll|ll} 
Parameter & Value & Parameter & Value & Parameter & Value \\
\hline Simulation network area & $1000 \times 1000 \mathrm{~m}^{2}$ & CCA threshold, $\Gamma$ & $-86 \mathrm{dBm}$ & PCS and RTS/CTS & Enabled \\
Slot-time & $20 \mu \mathrm{s}$ & STA Transmit power & $15.85 \mathrm{~mW}$ & AP Transmit Power & $100 \mathrm{~mW}$ \\
$N_{o}$ & $90 \mathrm{dBm}$ & AP Buffer Size & 20 Packets & P_REQ and P_RES Frames & 20 Bytes \\
CCA Time / SIFS & $15 \mu \mathrm{s} / 10 \mu \mathrm{s}$ & Receiver sensitivity, $\theta$ & $-90.96 \mathrm{dBm}$ & Mean Packet Size & 1460 bytes
\end{tabular}

consequently, any feasible $\left(\Psi_{j i}\right)_{i \in \mathcal{S}, j \in \mathcal{A}}$ that satisfies $\sqrt{7 \mathrm{~d})}$ also satisfies $(7 \mathrm{c})$ and renders $(7 \mathrm{c})$ redundant as well. Therefore, (7) can be equivalently expressed as:

$$
\begin{array}{ll}
\operatorname{maximize} & \sum_{j=1}^{M} \sum_{i=1}^{S} \mathbf{B}_{j i} x_{i j} \\
\text { subject to } & \sum_{j=1}^{M} x_{i j}=1, \forall i \in \mathcal{S}, x_{i j} \in\{0,1\} \\
& x_{i j} \Psi_{j i} \geq \gamma_{o}, \forall i \in \mathcal{S}, j \in \mathcal{A}
\end{array}
$$

The solution to (9) is given in Algorithm 2, where the problem is solved numerically using linear programming (LP). A typical STA $i$ captures SINR from all APs within range, then sets $\hat{\Psi}$ containing SINR through each candidate AP and uses the Gurobi LP solver [13] to locally determine its association, $x_{i j}=1$. The main goal of OPASA in Algorithm 2 is to serve as the optimal throughput benchmark given SINR constraints.

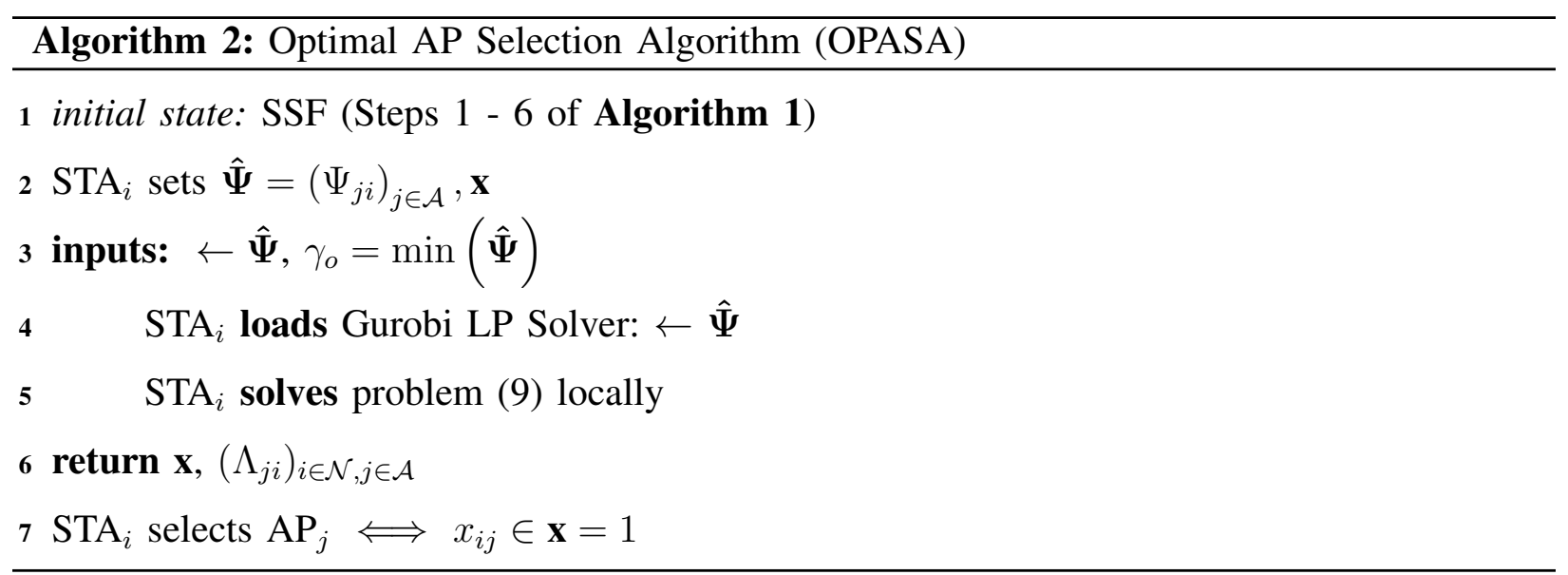




\section{PERFormance EVAluation}

This section presents the simulation methodology, scenario and results. For performance benchmarking, OPASA mainly serves as an optimal benchmark while DASA (Algorithm 1) is compared with the SSF scheme in 802.11 standards and the state-of-the-art mean probe delay (MPD) AP selection algorithm in [3].

\section{A. Simulation Setup and Parameters}

To simulate the channel access coordination at the MAC layer in a WiFi network, we have implemented the distribution coordination function (DCF) with a Slot-time of $20 \mu s$, short interframe space $(\mathrm{SIFS})=10 \mu \mathrm{s}, \mathrm{DIFS}=2 \times \mathrm{SIFS}$ and a CCA time of $15 \mu \mathrm{s}$ in MATLAB. The simulated network emulates a random AP deployment where APs and STAs are deployed on an area of $1000 \times 1000 \mathrm{~m}^{2}$. This network consists of 400 STAs and 50 APs deployed on three non-overlapping channels of IEEE $802.11 \mathrm{~b}$ PHY on a $2.4 \mathrm{GHz}$ band. All APs have identical coverage areas of $50 \mathrm{~m}$ radius and transmit with a uniform power of $100 \mathrm{~mW}(20 \mathrm{dBm})$. Table III summarizes other key parameters and the received power at $\mathrm{STA}_{i}$ from $\mathrm{AP} j$ is measured using $P_{j i}^{r}=P^{t}(\mathrm{~mW}) G_{j i} d_{j i}^{-\alpha}(\mathrm{dBm})$, where $G_{j i}$ is the channel gain characterized by an exponential distribution i.e. $G_{j i} \sim \exp \left(P^{t}\right)$ to account for fading and shadowing effects, and $\alpha=3$ is used as the path loss exponent. The minimum receiver sensitivity is set as $\theta=-90.96 \mathrm{dBm}$.

For carrier sensing, the PCS is enabled with minimum and maximum contention window (CW) sizes 32 and 1024, respectively, while request to send (RTS)/clear to send (CTS) frames are used to minimize the effect of the hidden terminal problem - a node does not transmit immediately after sensing the channel to be idle under PCS. Rather, it transmits the RTS frame and begins transmission of payload when the CTS frame is received. To emulate the asymmetric traffic requests in Wi-Fi networks, APs and STAs transmit packets of varying sizes (between 1400 to 1500 bytes) with a mean packet size of 1460 bytes while the MAC header, CTS/RTS frame and ACK frame sizes are 34, 14/20 and 14 bytes respectively as defined in the 802.11 standard. It is assumed that the $P_{-}$RES and $P_{-}$REQ frames have same size as the RTS. Packets arrive at each node's buffer at an exponential rate with parameter $\lambda=1 /$ Slot-time. 


\section{B. Simulation Results and Performance Benchmarking}

The primary performance metric is the aggregate downlink throughput. Figure 2 depicts the average achievable sum throughput for different network sizes. The duration of interference measurement in (2) is set as $T=n \times$ Slot time with $n=1000$. For the MPD, the probe delay is measured for the same duration and the AP with minimum probe delay is selected. From Figure 2, as expected, we infer that under any network size, DASA improves aggregate throughput. For instance, when the number of contending STAs is 300, DASA achieved $43 \%$ (43.22 to $61.84 \mathrm{Mbps}$ ) and 99\% (31.02 to $61.84 \mathrm{Mbps}$ ) throughput gains over MPD and SSF, respectively. With increasing network size, DASA outperforms existing MPD and SSF schemes with aggregate throughput approaching the optimal benchmark. Selecting AP with best DLSINR improves the PHY rate of each AP-to-STA link, which consequently improves aggregate end-to-end throughput.

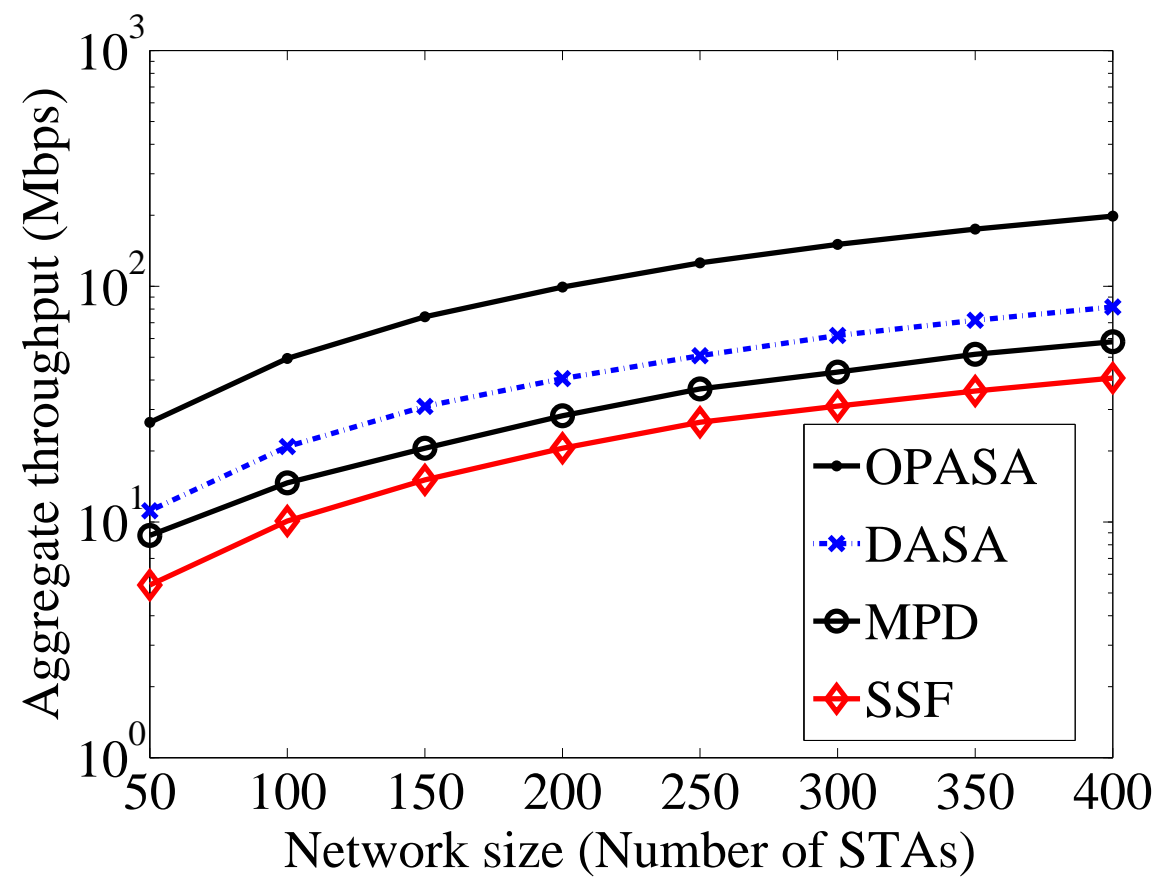

Fig. 2. Aggregate end-to-end throughput versus network size for $n=1000$ Slot times.

In Figure 3, the cumulative distribution of all STA throughputs is presented. Between 20th and 90th percentiles, DASA obtains higher throughput closer to the optimal OPASA than MPD and SSF. Observing the 40th percentile, the performance of MPD over SSF fluctuates while 


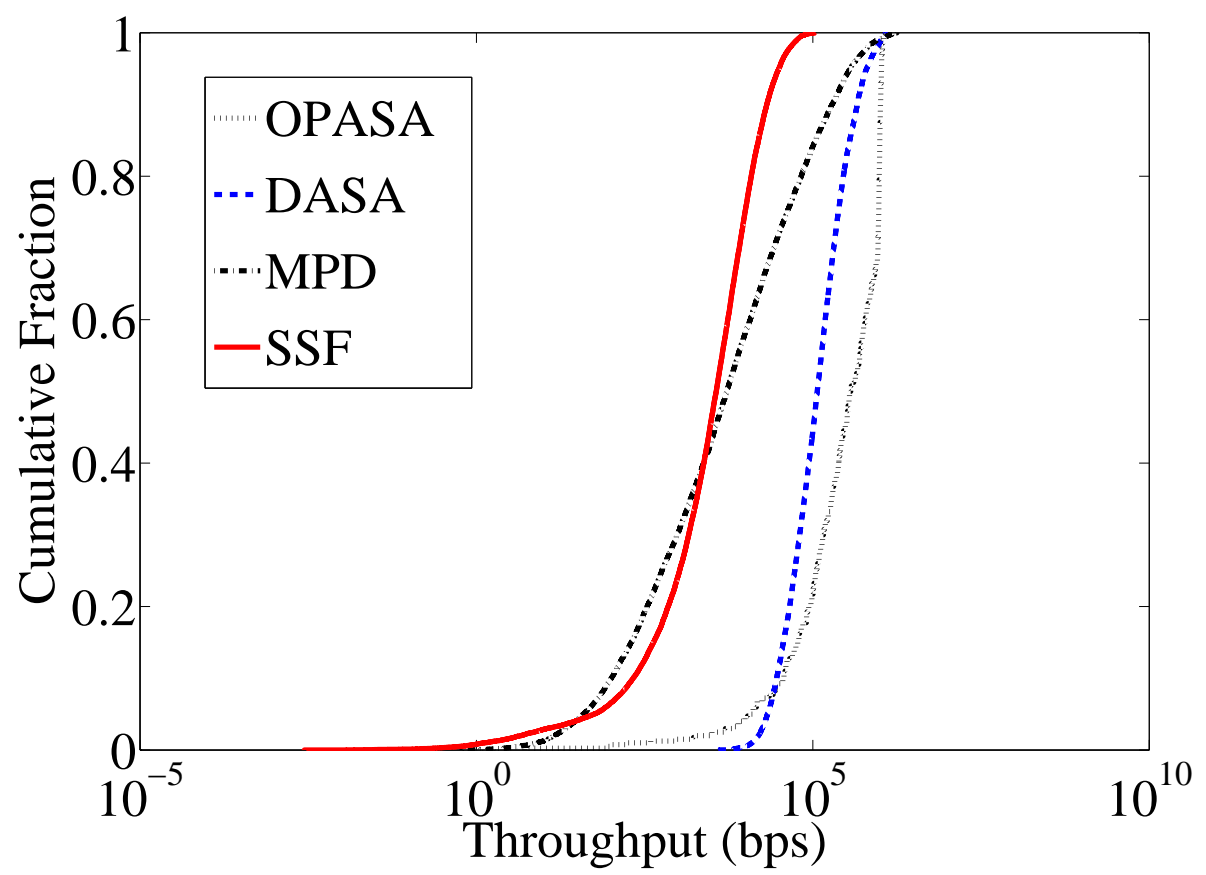

Fig. 3. Per-link throughput of 400 STAs for $n$ Slot-times, where $n=1000$.

DASA achieves nearly $2 \times$ gain over both SSF and MPD. At the 90th of the same Figure 3 , DASA maintains $5 \times$ gain over SSF while achieving 96.6\% gain over MPD. Between the 95 th and 100th percentile throughputs under MPD and DASA schemes converge.

Figure 4 illustrates end-to-end throughput of each of the 400 AP-to-STA links versus frame size. The first observation in Figure 4 is that as the frame size becomes larger, the throughputs achieved under MPD and DASA converge. This is likely due to the fact that delay becomes a factor in transmitting more bits and since MPD chooses links with less delay, more bits are likely to traverse the links at the same rate in DASA. Although, both MPD and DASA significantly outperform SSF, OPASA doubles the throughputs over DASA, SSF and MPD for frame sizes below and above 1485 bytes.

Figure 5 depicts the mean frame delay versus network size. Here, frame delay is the cumulative time from when a packet arrives at an AP's buffer and the AP contends for a channel, to successful reception of packet at the STA. For a small network size of $50 \mathrm{STAs}$, the delay is below $2 \mathrm{~ms}$ for SSF, MPD, OPASA and DASA while for larger network size of 400 users, the mean delay is higher in SSF. For 400 STAs under the SSF scheme, the average frame delay is nearly $9.19 \mathrm{~ms}$ 


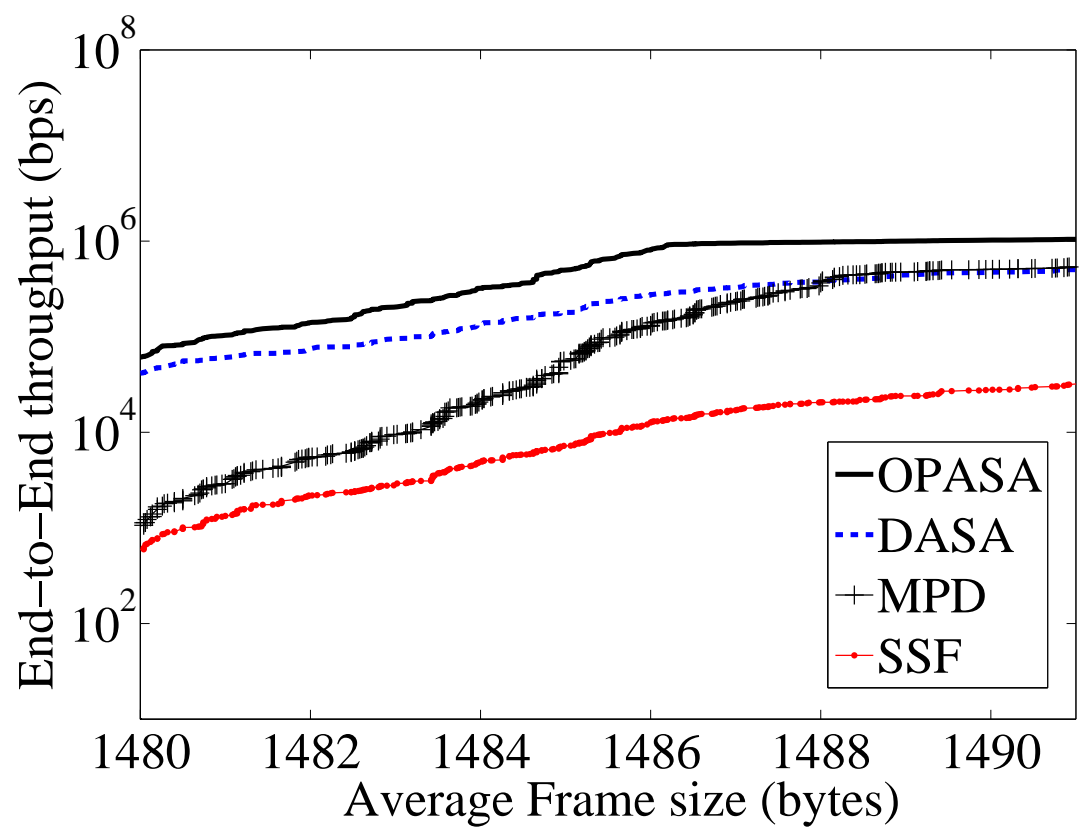

Fig. 4. End-to-end throughput of 400 STAs versus frame size.

while DASA and MPD maintain delays of $5.7 \mathrm{~ms}$ and $5.4 \mathrm{~ms}$, respectively. For lower network sizes (50 to 150 STAs), OPASA, MPD and DASA achieve nearly same performance in terms of delay, the slight superiority of MPD over OPASA and DASA becomes obvious when the network size increases from 200 to 400 STAs. This discrepancy is as a result of increased contentions among APs frequently trying to serve more STAs in the DL.

\section{CONCLUSION}

The problem of inter-BSS interference is inevitable in dense 802.11 networks and degrades performance. In fact, selecting an AP with strongest RSS does not always guarantee highest throughput due to interference at the target AP. We have shown that selecting AP based on SINR reduces the effect of interference among basic service sets (BSS). This paper presents a new scheme for AP-STA association that takes the AP interference into account. The OPASA algorithm serves as the optimal throughput benchmark while the much simpler proposed DASA algorithm provides significant gain in aggregate throughput while taking AP interference into account. Simulation results reveal that selecting the AP offering best SINR improves throughput. Through extensive simulation, the DASA algorithm is compared to the default SSF scheme used 


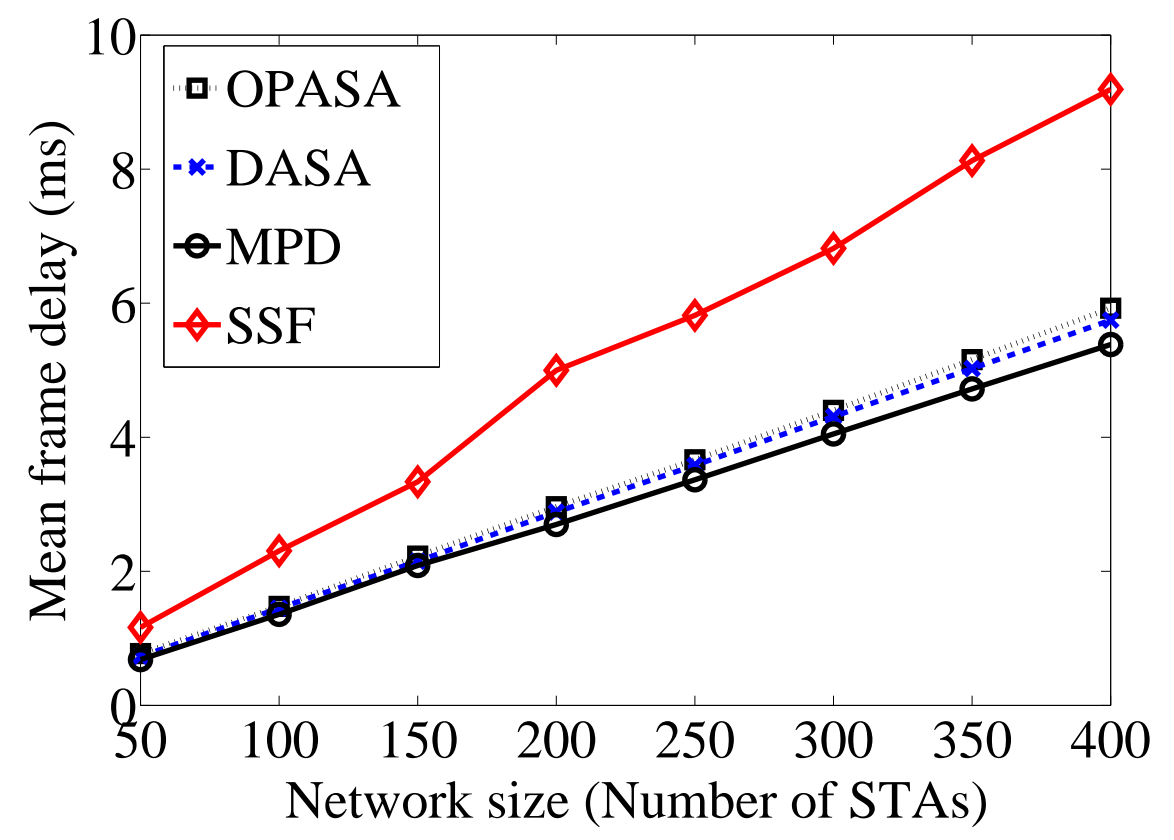

Fig. 5. Mean frame delay versus network size.

in current 802.11 standards and the MPD algorithm proposed previously; significant throughput gain over both the SSF and the MPD schemes is observed.

\section{REFERENCES}

[1] K. Lee et. al., "Mobile data offloading: how much can WiFi deliver?," in IEEE/ACM Trans. on Networking, vol. 21, no. 2, April 2013.

[2] K. Shin, I. Park, J. Hong, D. Har and D. Cho "Per-node throughput enhancement in Wi-Fi DenseNets," in IEEE Comm. Magazine, vol. 53,, no. 1, pp. 118 - 125, January, 2015.

[3] J. C. Chen et. al., "Effective AP selection and load balancing in IEEE 802.11 wireless LANs," in Proc. IEEE Globecom 2006.

[4] L. Wei et. al.,"AP association for proportional fairness in multirate WLANs," in IEEE/ACM Trans. on Net., vol. 22, no. 1, Feb., 2014.

[5] P. B. Oni and S. D. Blostein "AP association optimization and CCA threshold adjustment in Dense WLANs," in Proc. IEEE Globecom 2015 Workshop on Enabling Tech. in Future Wirel. Local Area Net., 2015.

[6] K. Masahiro, et al. "A trigger-based dynamic load balancing method for WLANs using virtualized network interfaces," in Proc. WCNC, 2013.

[7] D. Lei, et. al., "QoS aware access point selection for pre-load-balancing in multi-BSSs WLAN," in Proc. WCNC, 2008.

[8] D. Lei, B. Yong and C. Lan "Access point selection strategy for large-scale wireless local area networks," in Proc. WCNC, 2007. 
[9] K. Hong, et al. "Channel measurement-based access point selection in IEEE 802.11 WLANs," in Pervasive and Mobile Computing, 2015.

[10] Bojovic B, et. al., "A supervised learning approach to cognitive access point selection” IEEE Globecom Workshops, 2011.

[11] F. Xu, et. al., "SmartAssoc: decentralized access point selection algorithm to improve throughput," in IEEE Trans. on Parallel Distrib. Sys., vol. 24, no. 12, Dec., 2013.

[12] A. Murad, "A new approach for interference measurement in 802.11 WLANs," 21st Annual IEEE Int'l Symp. on PIMRC., 2010.

[13] Gurobi, “Gurobi Optimization,” http://www.gurobi.com Accessed: March 2nd, 2014. 